\title{
Pyrosequencing reveals shifts in the bacterial epimural community relative to dietary concentrate amount in goats
}

\author{
S. U. Wetzels, ${ }^{\star} † \ddagger$ E. Mann, $\ddagger$ B. U. Metzler-Zebeli, $¥ \S$ M. Wagner, $\ddagger$ F. Klevenhusen, ${ }^{\star} \ddagger$ Q. Zebeli, ${ }^{\star} \ddagger$ \\ and S. Schmitz-Essertł ${ }^{1}$ \\ *Institute of Animal Nutrition and Functional Plant Compounds, \\ †Institute for Milk Hygiene, Milk Technology and Food Science, \\ $\ddagger$ Research Cluster Animal Gut Health, and \\ §University Clinic for Swine, Department for Farm Animals and Veterinary Public Health, University of Veterinary Medicine Vienna, \\ 1210 Vienna, Austria
}

\section{ABSTRACT}

Ecological balance in the rumen is highly sensitive to concentrate-rich diets. Yet the effects of these feeding practices on the caprine bacterial epimural microbiome (CBEM), a microbial community with putative important physiological functions in the rumen, are largely unexplored. This study aimed to investigate the effect of dietary concentrate amount on ruminal CBEM. Seventeen growing goats were fed diets with $0[\mathrm{n}=5 ; 6.2$ MJ of metabolizable energy $(\mathrm{ME}) / \mathrm{d}], 30(\mathrm{n}=6 ; 7.3$ MJ of $/ d$ ), or $60 \%$ ( $\mathrm{n}=6 ; 10.2 \mathrm{MJ}$ of $\mathrm{ME} / \mathrm{d})$ concentrate for 6 wk. Two hours after their last feeding, goats were euthanized and tissue samples of the ventral rumen wall were collected, washed in phosphate-buffered saline to detach loosely attached bacteria, and stored at $-20^{\circ} \mathrm{C}$ for further processing. Genomic DNA was isolated from thawed rumen mucosa samples and used for Roche/454 Life Science (Branford, CT) 16S rRNA gene amplicon pyrosequencing yielding 122,458 reads. Pyrosequencing data were clustered into 1,879 operational taxonomic units (OTU; 0.03 distance level). Pyrosequencing revealed Proteobacteria, Bacteroidetes, Firmicutes, and Spirochaetes as the most abundant phyla $(97.7 \%)$. Compared with the $30 \%$ group, both the 60 and $0 \%$ concentrate groups harbored significantly more Firmicutes and SR1, respectively. On an OTU level, a Bergeriella-related OTU was most abundant in the CBEM, followed by 2 Campylobacter OTU, which responded differently to diets: 1 OTU was significantly increased whereas the other significantly decreased with highest concentrate amount in the diet. At the genus level, the $0 \%$ concentrate group harbored increased Kingella-like sequences compared with the other feeding groups. Furthermore, the $0 \%$ concentrate group tended

Received November 27, 2014.

Accepted April 19, 2015.

${ }^{1}$ Corresponding author: Stephan.Schmitz-Esser@vetmeduni.ac.at to have more Bergeriella than the 30 and $60 \%$ concentrate groups. The genus Bergeriella was significantly decreased in the $60 \%$ feeding group compared with the other diets. In conclusion, this is the first report of CBEM using deep-sequencing methods on the genus and OTU level, and our study revealed major shifts in the CBEM in response to concentrate-rich diets with potential health relevance in goats.

Key words: rumen epithelium, microbiota, subacute rumen acidosis, goat, feeding

\section{INTRODUCTION}

The gastrointestinal tract of ruminants is a highly complex and mostly anaerobic ecosystem. Trillions of microorganisms, including bacteria, archaea, ciliate protozoa, fungi, and viruses interact or compete in a symbiotic, commensal, or potentially pathogenic way with the host (Hungate, 1966; Luckey, 1972; Kamra, 2005). In the rumen, bacteria are the predominant microorganisms, fermenting largely indigestible plant biomass to products such as short-chain fatty acids (SCFA), an essential energy source for the host ruminant (Bergman, 1990; Mackie, 2000). The ruminal mucosa-associated (epimural) bacterial fraction performs a multitude of symbiotically relevant functions necessary for ruminants' overall metabolism and health (Sadet et al., 2007; Chen et al., 2011; Li et al., 2012). Early cultivation-dependent and -independent studies revealed a distinct bacterial community pattern between the rumen content and the epimural site (Cheng and Wallace, 1979). Due to the higher absolute number of bacteria, the metabolic activity of luminal bacteria is more substantial compared with epimural bacteria. Nevertheless, the bacterial epimural microbiome is the primary contact line to the mucosal sites of the rumen. Moreover, epimural bacteria competitively exclude with adhesive and putative pathogenic microorganisms and provide protection against harmful microbiota by, for example, forming a protecting biofilm on the ruminal 
epithelium (Leser and Molbak, 2009; Sadet-Bourgeteau et al., 2010; Chen et al., 2011).

Among the first groups to cultivate bacteria from caprine rumina, Dehority and Grubb (1977) described up to $70 \%$ of the cultivated bacteria classified as $\mathrm{Bu}$ tyrivibrio affiliating to the class Clostridia. Although cultivation-dependent methods underestimate bacterial diversity in the rumen (Hungate, 1966; Cheng and Wallace, 1979; Amann et al., 1995), Clostridia, together with Bacteroidia, have also been described recently for being predominant in the caprine rumen by cloning and sequencing 16S rRNA genes (Cunha et al., 2011). More recently, Lee et al. (2012) used pyrosequencing for the characterization of the microbiome in the goat rumen content and described it as clearly distinct from those of other ruminants. However, the literature lacks information about the caprine bacterial epimural microbiome (CBEM) and little is known about how it adapts to dietary changes (Huo et al., 2014; Han et al., 2015; Liu et al., 2015). This makes it difficult to predict adaptive responses of the CBEM in the rumen. In contrast to cattle and sheep, the goat is a browser ruminant, adapted to thrive under low-quality forage dietary conditions. However, the intensive goat production systems dictate feeding of high-energy diets. Consequently, the roughage-based feeding practices have been consistently replaced by concentrate-rich diets in intensive goat farming. These shifts in feeding practices typically result in a decrease in ruminal $\mathrm{pH}$, increase of osmolarity, change in composition of the SCFA and substrates available for rumen microbes, and changes in fermentation processes (Khafipour et al., 2009). The accumulation of these events could lead to SARA, a severe rumen metabolic disorder in ruminants (Fernando et al., 2010). Indeed, SARA affected the diversity and community structure of rumen liquid and solid associated bacteria in goats (Huo et al., 2014). We recently fed goats diets based on hay ( $0 \%$ concentrate) and 30 or $60 \%$ concentrate and observed major changes in electrophysiological properties and permeability (Klevenhusen et al., 2013), greater keratinization, and differences in the abundance of luminal microbial populations and LPS, as well as depletion of cellular protective factors of the rumen epithelium (Hollmann et al., 2013; Metzler-Zebeli et al., 2013a,b). Because of the inherent relationship of CBEM with rumen epithelial lining, we hypothesized that feeding of high-concentrate diets and the resulting acidotic challenge in the rumen would also result in shifts in the community structure of CBEM. Major aims of our study were to define the core CBEM and to investigate shifts in the CBEM in response to 3 different levels of energy supply in the diet using Roche/454 Life Science (Branford, CT) pyrosequencing of $16 \mathrm{~S}$ rRNA gene amplicons.

\section{MATERIALS AND METHODS}

\section{Animals, Diets, and Experimental Design}

The animal experimental protocol of this research was discussed and approved by the institutional ethics committee of the University of Veterinary Medicine Vienna in accordance with Good Scientific Practice guidelines and national legislation (Protocol no. 15/03/97/2011). The experiment was performed at the experimental station of the Institute for Organic Farming and Biodiversity in Wels, Austria. Experimental animals were the same as in previous studies where rumen digesta microbiota and rumen and colon epithelial host response were examined (Hollmann et al., 2013; Klevenhusen et al., 2013; Metzler-Zebeli et al., 2013a,b). In Supplementary Table S1 (http://dx.doi.org/10.3168/jds.2014-9166), relevant results from previous publications outside the current study are summarized. Seventeen goats, 4 mo of age, were used in our study. Before the experiment started, the goats were fed a high-forage diet containing $1.5 \mathrm{~kg}$ of chopped second-cut meadow hay (88\% DM, $11.2 \% \mathrm{CP}, 8.4 \%$ ash, $55.6 \% \mathrm{NDF}$ ) as forage and $200 \mathrm{~g}$ of coarsely ground barley concentrate $(88.3 \%$ DM, $3.4 \%$ ash, $13 \%$ CP, $20.5 \%$ NDF; Lagerhaus, Wels, Austria) daily per animal. The animals were randomly allocated to 1 of the 3 experimental diets differing in their concentrate amount. The groups were balanced for BW and breed. Diets and feeding procedure have been explained in detail previously (Metzler-Zebeli et al., 2013a). Briefly, the control goats received a diet composed purely of hay (6.2 MJ of $\mathrm{ME} / \mathrm{d}$ ) without any concentrate $(0 \%$ concentrate diet). The medium (7.3 $\mathrm{MJ}$ of $\mathrm{ME} / \mathrm{d}$ ) and high (10.2 MJ of ME/d) concentrate diets consisted of hay plus 30 and $60 \%$ concentrate (coarsely ground barley, a rapidly fermentable grain) on a DM basis, respectively. The hay and concentrate fed to the goats originated from 1 batch and the goats were adapted to the 30 and $60 \%$ concentrate diets by increasing the concentrate level step-by-step by 2.5 and $5 \%$ per day, respectively. Barley grain was selected as concentrate due to its rapid degradation in the rumen combined with a high potential to cause acidotic conditions in the rumen. The experiment started after all goats reached the 30 and $60 \%$ concentrate level. The animals were fed separately, twice a day at 0630 and $1330 \mathrm{~h}$, and were weighed weekly for adjusting the feed allowance. In total, the feeding experiment lasted $6 \mathrm{wk}$.

\section{Rumen Mucosa Sampling}

To investigate shifts in the CBEM, the goats were euthanized 2 to $3 \mathrm{~h}$ after the morning feeding on the last day of the feeding experiment (d 42) by an intravenous 
injection of Release (50 mg of pentobarbital $/ \mathrm{kg}$ of BW; WDT, Garbsen, Germany) and Xylosol (0.5 mg of xylazine/kg of BW; Ogris Pharma, Wels, Austria). After opening the abdominal cavity, the rumen was carefully removed and opened from the dorsal side. The rumen was emptied and a $4-\times 4$-cm piece of the ventral rumen wall tissue was cut and placed into $100 \mathrm{~mL}$ of sterile $1 \times$ PBS (room temperature) for transferring to the laboratory. In the laboratory, the rumen wall was rigorously washed in $100 \mathrm{~mL}$ of $1 \times$ PBS (this procedure was done 3 times) to detach the free and loosely attached bacteria. The samples were cut into $1-$ to $2-\mathrm{cm}^{2}$ pieces and stored in $1 \times \mathrm{PBS}$ at $-20^{\circ} \mathrm{C}$ for further processing.

\section{DNA Extraction}

The rumen mucosa samples were thawed on ice and genomic DNA was extracted from $0.25 \mathrm{~g}$ of rumen mucosa (cut in $2-\times 2-\mathrm{mm}$ pieces), using the PowerSoil DNA Isolation Kit (MO BIO Laboratories Inc., Carlsbad, CA) according to the manufacturers protocol (http://www.mobio.com), with 1 additional step (after the first vortexing for $10 \mathrm{~min}$, the sample was ground by rotating the mortar 10 times to the left and to the right, followed by $5 \mathrm{~min}$ of vortexing again). The DNA concentration was quantified using the Qubit 2.0 Fluorimeter (Thermo Fisher Scientific, Vienna, Austria).

\section{Preparation of 16S rRNA Gene Amplicon Libraries, Pyrosequencing and Processing, and Taxonomic Assignment of Sequence Reads}

The DNA samples were sequenced at the Center for Medical Research Medical University in Graz (Austria) using a 454 GS FLX Titanium Sequencing System (LibL kit primer A-primer B; Roche/454, Life Science). The 16S rRNA genes were amplified using the universal primers F27 (forward $=$ 5'-AGA GTT TGA TCC TGG CTC AG-3'; Microsynth, Balgach, Switzerland) and R357 (reverse $=5^{\prime}$-CTG CTG CCT YCC GTA-3'; Microsynth) targeting the V1-V2 hypervariable regions of the $16 \mathrm{~S}$ rRNA gene. The $25-\mu \mathrm{L}$ PCR reaction, performed in triplicate, contained $1 \times$ fast start buffer; 1.25 $\mathrm{U}$ of high-fidelity enzyme; $200 \mu M$ (each) dATP, dTTP, dGTP, and dCTP; $0.4 \mu M$ barcoded primers (Eurofins MWG, Ebersberg, Germany); $2.5 \mathrm{mM} \mathrm{MgCl}_{2}$; and PCR-grade water (Roche Diagnostics, Mannheim, Germany). Total genomic DNA (100 ng) was added to the PCR. Amplification in a standard thermocycler after initial denaturation at $95^{\circ} \mathrm{C}$ for 3 min was performed in 30 cycles at $95^{\circ} \mathrm{C}$ for $45 \mathrm{~s}$, annealing at $55^{\circ} \mathrm{C}$ for $45 \mathrm{~s}$, and extension at $72^{\circ} \mathrm{C}$ for $1 \mathrm{~min}$ with a final extension for 7 min. Amplicons were purified using a WAVE Sys- tem (Transgenomic Inc., Omaha, NE) and eluted by a linear gradient of acetonitrile in $0.1 M$ trimethyl ammonium acetate; amplicon DNA was purified subsequently on NucleoFast 96 PCR plates (Macherey-Nagel, Düren, Germany). The DNA concentrations were determined using a PicoGreen double-stranded DNA assay kit (Life Technologies, Carlsbad, CA). Barcode-labeled amplicons were pooled equimolar and analyzed using a 2100 Bio Analyzer (Agilent Technologies, Waldbronn, Germany) using a DNA 7500 kit. Emulsion PCR of pooled samples was performed with the GS Titanium MV emPCR kit (Roche/454 Life Science) according to manufacturer's instructions. Sequencing of the pool was performed using the GS FLX Titanium Sequencing Kit XLR70 (Roche/454 Life Science) according to manufacturer instructions.

Analysis of the sequence data (17 samples; 122,458 reads in total) was done with the software package mothur (version 1.33.3, http://www.mothur.org/) according to the procedure described previously (Schloss et al., 2009; Schloss and Westcott, 2011). Primers, barcode sequences, and sequences of short length and low quality were trimmed with a minimum average quality score of 30 and a minimum length of $145 \mathrm{bp}$. The command "Chimera.uchime" was used to exclude chimeric sequences. A total of 82,047 sequences $(67 \%)$ passed the quality control. The sequences were clustered into operational taxonomic units (OTU) using $97 \%$ 16S rRNA gene similarity as a threshold (0.03 distance; Kuczynski et al., 2012). The OTUs containing only 1 sequence were removed (2,363 OTU). For calculating the nonparametric estimators, Chao 1 and ACE, and the diversity indices, Shannon, Simpson, and Bray-Curtis similarity, the "summary.single" command was used after rarefying to the minimum number of sequences per sample. Rarefaction curves were generated and modified after an algorithm described by (Griffen et al., 2012). Heatmaps were created using JcolorGrid (http://jcolorgrid.sourceforge.net/; Joachimiak et al., 2006). To illustrate microbial shifts on community level, linear discriminant analysis was done in JMP Pro (SAS Institute, Cary, NC) with the 45 most-abundant OTU as covariates and diet as the categorical variable. Cluster analysis was done with Cluster 3.0 (Clustering Library version 1.52; de Hoon et al., 2004). The OTUs that were detected in all feeding groups $(\mathrm{n}=322)$ were normalized and k-means clustering was performed. In the heatmap, Euclidean distances (median values) are visualized in Java Treeview (Saldanha, 2004).

Diet-group-specific prediction of the metagenomic potential of the CBEM was performed with PICRUSt, based on 16S rRNA data (Phylogenetic Investigation of Communities by Reconstruction of Unobserved States; 
Langille et al., 2013). This tool is based on the assumption that phylogeny can reliably predict gene content. For PICRUSt, another OTU picking was performed with quality controlled sequences by aligning sequences to Greengenes reference OTUs (downloaded from http://greengenes.secondgenome.com/downloads/database/13_5) and OTUs were clustered based on 0.03 distance limit. This OTU table was used for PICRUSt on the online Galaxy interface (http://galaxyproject. org/), with a workflow described by the developers (http://picrust.github.com/picrust/tutorials/quickstart.html\#quickstartguide).

\section{Cloning and Sanger-Sequencing for Comparison with Pyrosequencing Data}

The PCR amplification of $16 \mathrm{~S}$ rRNA genes were done with primers $616 \mathrm{~F}$ (forward $=5^{\prime}$-AGA GTT TGA TYM TGG CTC-3'; Juretschko et al., 1998) and 1492R (reverse $=5^{\prime}$-GGY TAC CTT GTT ACG ACT T-3'; Lane, 1991). Reaction mixtures (total volume $=25 \mu \mathrm{L}$ ) containing each primer at a concentration of $0.2 \mathrm{pmol} /$ $\mu \mathrm{L}$ were prepared by using $1 \times$ reaction buffer $(\mathrm{Ex}$ Taq Buffer; $20 \mathrm{mM} \mathrm{MgCl}$ ) and $0.025 \mathrm{U}$ of TaKaRa Ex Taq Hot Start polymerase (TaKaRa 5 units/ $\mu \mathrm{L}$ ), $0.8 \mathrm{mM}$ (0.2 $\mathrm{m} M$ each) of dNTP (TaKaRa, Shiga, Japan), and $0.5 \mu \mathrm{L}$ of template DNA. Thermal cycling was carried out by using an initial denaturation step of $95^{\circ} \mathrm{C}$ for 5 min, followed by 25 cycles of denaturation at $98^{\circ} \mathrm{C}$ for $10 \mathrm{~s}$, annealing at $52^{\circ} \mathrm{C}$ for $40 \mathrm{~s}$, and elongation at $72^{\circ} \mathrm{C}$ for $60 \mathrm{~s}$. Cycling was completed by a final elongation step of $72^{\circ} \mathrm{C}$ for $5 \mathrm{~min}$. The StrataClone PCR Cloning Kit (Agilent, Santa Clara, CA) was used for cloning following the manufacturer's instructions. In total, 328 clones were sequenced by Sanger sequencing (LGC Genomics, Berlin, Germany) using vector-specific primers. Overlapping sequences were assembled and vector sequences were removed using the DNA Baser software (http://www.dnabaser.com/). Comparison of pyrosequencing OTUs and cloning OTUs was performed with local BLASTn searches (https://blast.ncbi.nlm.nih. gov/Blast.cgi). Each of the near full-length sequences showing $\geq 99 \%$ similarity to pyrosequencing OTU were considered fitting. Sanger sequencing OTUs were classified against type strains using RDP (Ribosomal Database Project; http://rdp.cme.msu.edu/index.jsp).

\section{Accession Numbers}

The pyrosequencing data are available in the European Nucleotide Archive under accession number PRJEB7404. The clone sequences are available in the European Nucleotide Archive under accession numbers LN612774-LN613101.

\section{Statistical Analyses}

Data were first analyzed for normality using ShapiroWilk test with PROC UNIVARIATE in SAS (version 9.2, SAS Institute Inc.). The parametric analysis of the data was conducted using PROC MIXED of SAS. Data were submitted to ANOVA with a model accounting for the fixed effects of feeding group and random effect of animal. Polynomial contrasts were used to evaluate linear and quadratic effects of concentrate inclusion and to contrast the effects of 0 versus 30 and $60 \%$ grain and 0 and 30 versus $60 \%$ concentrate. Degrees of freedom were estimated using the method of Kenward-Roger. Significance was defined as $P \leq 0.05$ and trends were defined at a level of $0.05<P \leq 0.10$. Results are shown as least squares means \pm standard error of the mean.

\section{RESULTS}

\section{Pyrosequencing Data and OTU Classification}

All 122,458 reads deriving from 17 samples were processed together throughout the bioinformatics workflow. In total, 82,047 sequences $(67 \%)$ with a minimum length of $145 \mathrm{bp}$ passed the quality control $(4,357 \pm$ 855 sequences per sample). Sequences were clustered into 4,242 OTU, from which 2,363 OTU were excluded because they consisted of only 1 sequence. The remaining 1,879 OTU were used for all further downstream analyses. Each of the 12 most abundant OTU contained >1,000 sequences (up to 10,364 sequences; Supplementary Table S2; http://dx.doi.org/10.3168/ jds.2014-9166).

\section{Assessment of Diversity and Surveys of CBEM Structure}

Rarefaction curves (Figure 1A) were calculated for all samples and summarized to diet means to determine the coverage of sequenced diversity per group. Curves revealed sufficient high diversity coverage for all feeding groups. Rank abundance curves (Figure 1B) indicated that the samples contained a low proportion of highly abundant OTUs, whereas most of the present diversity was composed of rare organisms. Diversity indices estimating species richness and evenness are shown in Table 1. Diversity indices were not different among feeding groups $(P>0.1)$. The actual numbers of OTUs found in each feeding group were 851, 1,129, and 1,179 in the 0,30 , and $60 \%$ concentrate diets, respectively.

As shown in Figure 1, 323 OTU (17.2\%) were shared among the 3 feeding groups, whereas 634 OTU $(33.8 \%)$ could be detected in 2 out of 3 feeding groups, and 922 OTU (49\%) were detected in only 1 of the feeding 

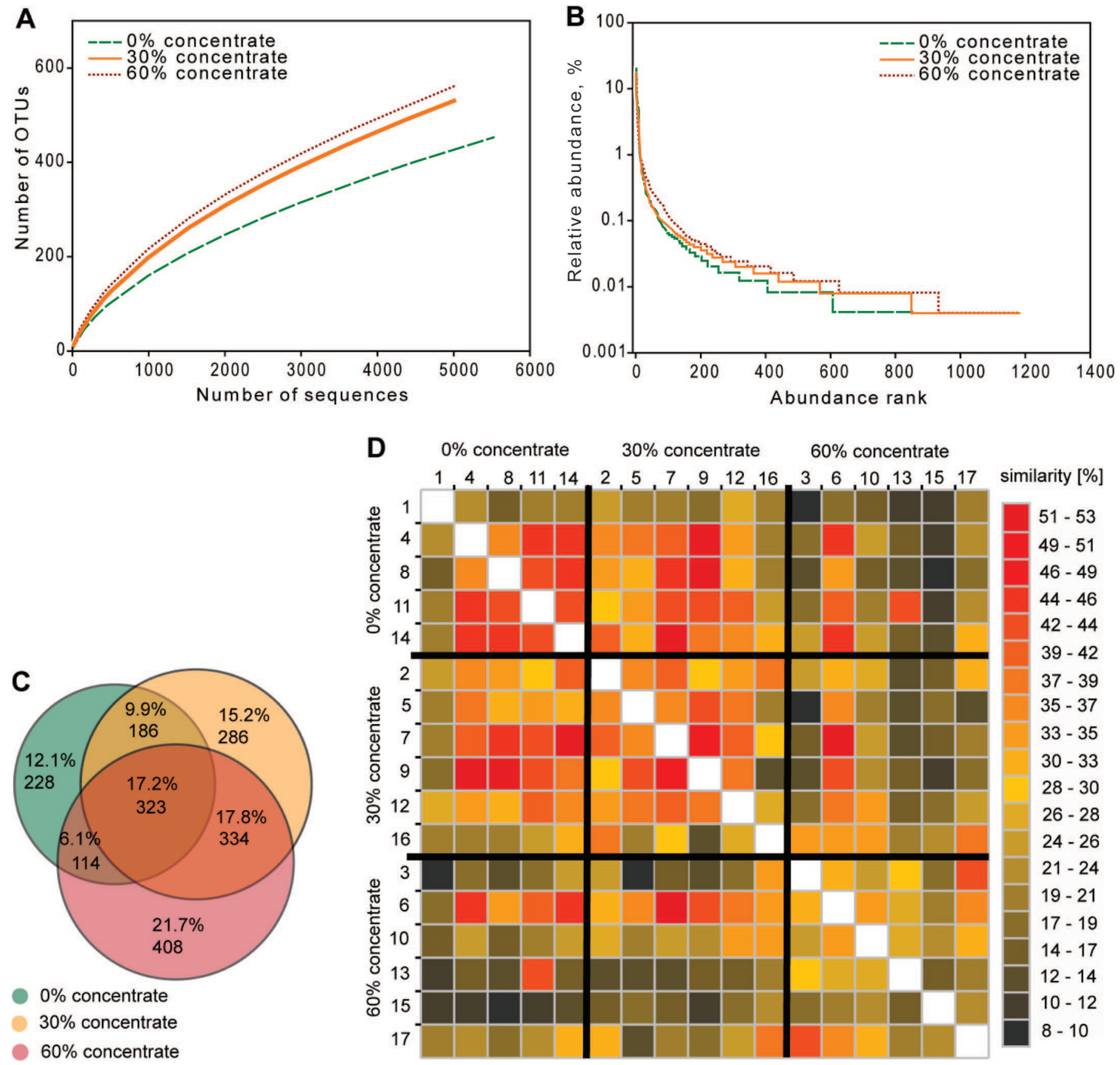

Figure 1. Diversity of bacteria attached to the rumen wall. (A) Rarefaction curves and (B) rank abundance curves based on an operational taxonomic unit (OTU) definition threshold of $0.0316 \mathrm{~S}$ rRNA distance are shown. Rarefaction and rank abundance curves were calculated for each sample and depicted as mean values per feeding group. (C) Venn diagram showing the number of shared OTU between feeding groups. The size of the circle is in proportion to the number of OTUs detected in each feeding group. (D) Bray-Curtis analysis displays 16S rRNA gene similarity results between the 3 feeding groups $(0,30$, and $60 \%$ barley concentrate) and the single goats compared with each other. Color version available online.

groups (Figure 1C). On a community level, shown by Bray-Curtis similarities, the comparison of 0 and $30 \%$ concentrate groups showed relatively high similarity scores (between 30 and 53\% similarity), except for goat 1 compared with all other goats. In contrast, the $60 \%$ concentrate group (with the exception of goat 6) displayed only low similarity to the other 2 feeding groups as well as low similarity within the $60 \%$ concentrate group (Figure 1D). However, pairwise comparisons of the microbial communities from different feeding 
Table 1. Species richness and diversity indices for bacteria attached to the rumen wall by estimator ${ }^{1}$

\begin{tabular}{lcccc}
\hline Feeding group & Chao 1 & $\mathrm{ACE}^{2}$ & Shannon & Simpson \\
\hline $0 \%$ concentrate & 713 & 1,070 & 3.34 & 0.11 \\
SEM & $(662-1,003)$ & $(1,063-1,360)$ & $(3.69-3.84)$ & $(0.08-0.09)$ \\
$30 \%$ barley concentrate & 103 & 157 & 0.38 & 0.02 \\
& 816 & 1,235 & 3.79 & 0.08 \\
SEM & $(662-1,003)$ & $(1,063-1,360)$ & $(3.69-3.84)$ & $(0.08-0.09)$ \\
$60 \%$ barley concentrate & 94 & 143 & 0.082 & 0.02 \\
& 855 & 1,269 & 4.09 & 0.06 \\
SEM & $(662-1,003)$ & $(1,063-1,360)$ & $(3.69-3.84)$ & $(0.08-0.09)$ \\
\hline
\end{tabular}

${ }^{1}$ Values of richness and diversity indices did not differ significantly among feeding groups. Values in parentheses represent confidence intervals.

${ }^{2} \mathrm{ACE}=$ abundance-based coverage estimator.

groups by using libshuff analysis revealed that all feeding groups were statistically distinct from each other (data not shown).

\section{Phylum Affiliation}

In total, 23 phyla were identified and Proteobacteria, Bacteroidetes, Firmicutes, and Spirochaetes were the most abundant, with $97.7 \%$ of all reads affiliating to these 4 phyla. In Figure 2 and Supplementary Table S3 (http://dx.doi.org/10.3168/jds.2014-9166), relative abundances of the phyla are depicted with respect to the feeding groups. There was a trend of decreasing Proteobacteria $(P=0.053)$ in the $60 \%$ concentrate group compared with the other feeding groups. A significant increase of Firmicutes $(P=0.041)$ in the $60 \%$ concentrate group and $S R 1(P=0.034)$ in the $0 \%$ concentrate group could be detected compared with the other feeding groups. A trend was also observed for a decreasing average number of phyla in the $60 \%$ concentrate group compared with the other 2 feeding groups $(P<0.08)$.

\section{Diet-Related Shifts on the OTU Level}

Linear discriminant analysis with diet as the categorical variable revealed strong differences among the feeding groups in response to concentrate content of the diet. All feeding groups clustered separately, with no overlapping in their 95\% confidence intervals (Figure
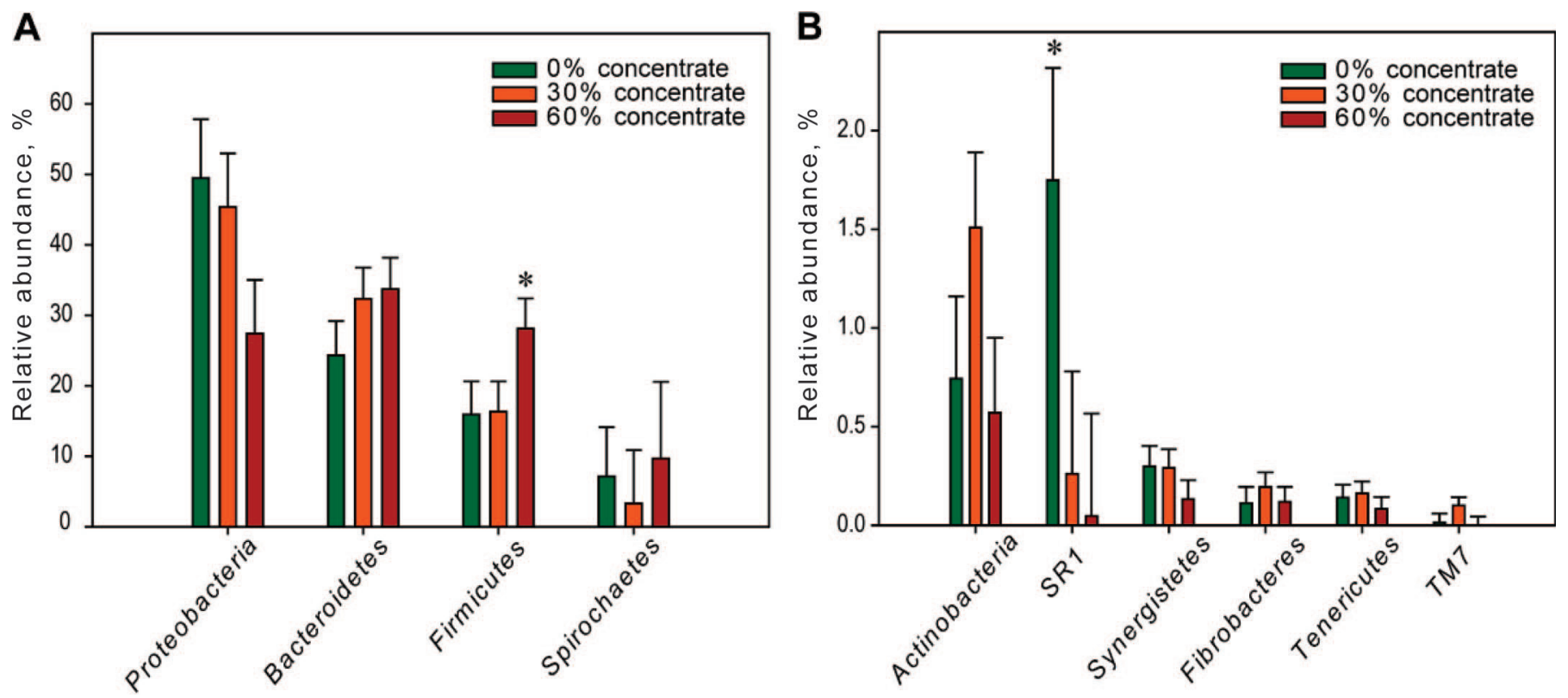

Figure 2. Relative abundances of the 10 most-abundant bacterial phyla attached to the rumen wall. (A) Abundant phyla with more than $2 \%$ mean abundance are shown for each feeding group (0,30, and $60 \%$ barley concentrate). (B) Relative abundances of rare phyla for each feeding group. Error bars represent SEM. Significance in one feeding group compared with the other 2 feeding groups are marked with an asterisk (*) and defined as $P \leq 0.05$. Color version available online. 
3). Relative abundances of the 45 most abundant OTU are shown in Figure 4 and listed in detail in Supplementary Tables S2 and S4 (http://dx.doi.org/10.3168/ jds.2014-9166). In total, the 45 most abundant OTU accounted for $73.8 \%$ of all sequences, whereas $28.9 \%$ of the 45 most abundant OTU $(\mathrm{n}=13)$ differed significantly between the feeding groups.

In the $0 \%$ concentrate group, only 5 OTU were highly abundant (relative abundance $>5 \%$ ), classified as Bergeriella, Campylobacter, Prevotella, and Meniscus. Ottowia-OTU 14 was significantly decreased $(0.2$ fold-change, $P<0.05$ ) and OTU 20 (unclassified SR1) was significantly increased (12.78 fold-change, $P<$
0.04 ) in the $0 \%$ concentrate group compared with the other feeding groups. In the $30 \%$ concentrate group, only 3 OTU were highly abundant (relative abundance $>5 \%$ ) and classified as Bergeriella, Campylobacter, and Prevotella, the 3 most abundant genera in our study across all feeding types. In the $60 \%$ concentrate group, 4 OTU were highly abundant (relative abundance $>5 \%$ ). Interestingly, Campylobacter-OTU 2 was significantly increased (2.89 fold-change, $P<0.02)$ in the $60 \%$ concentrate group, whereas Campylobacter-OTU 3 is significantly decreased ( 0.25 fold-change, $P<0.03)$ in the $60 \%$ concentrate group compared with the other feeding groups.

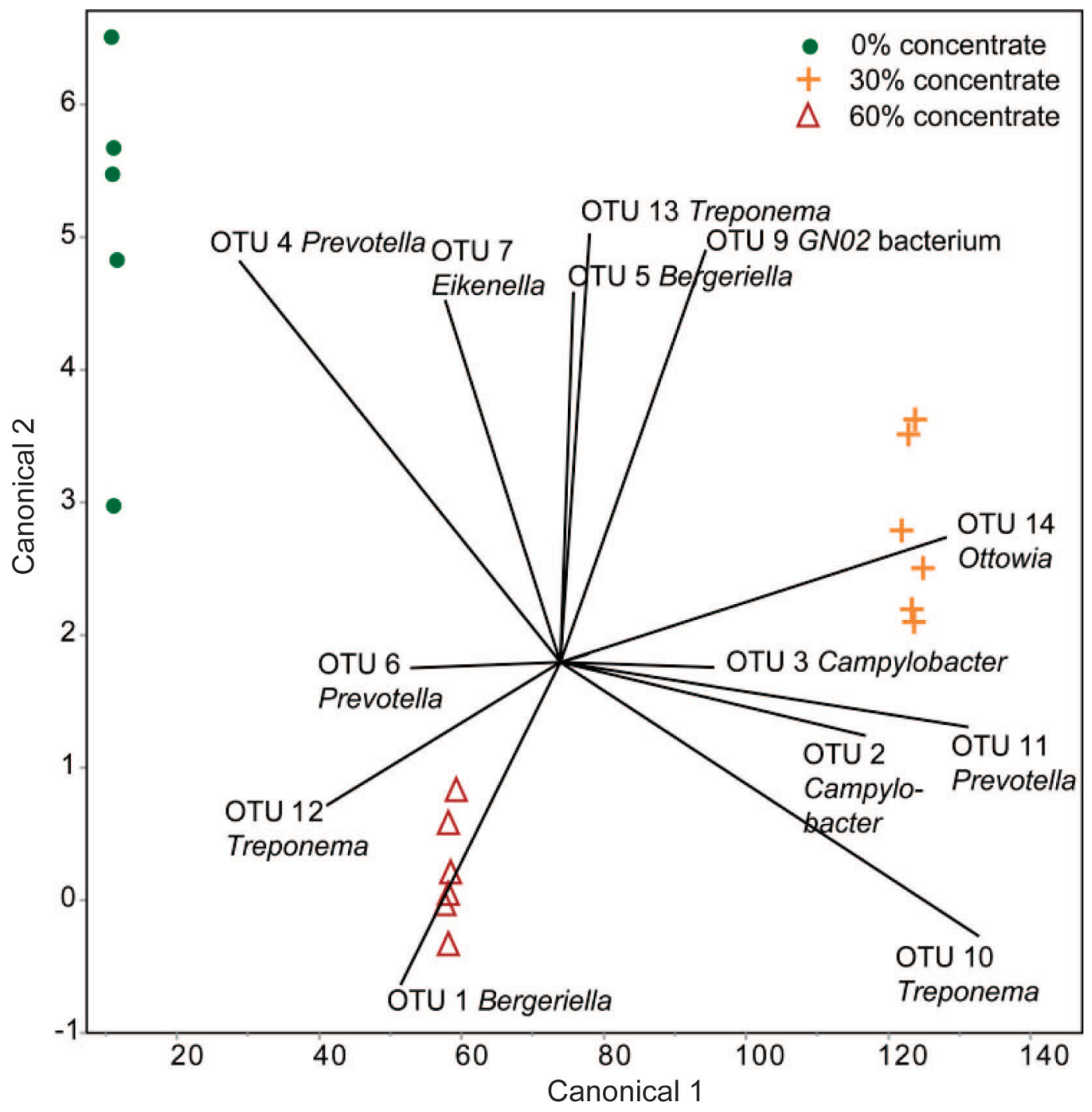

Figure 3. Linear discriminant analysis with the first 2 principal components for the epimural bacteria dependent on 3 different diets. For the discriminant analysis the 45 most-abundant operational taxonomic units (OTU) (representing $73.8 \%$ of all reads) were used as covariates and the diet as the categorial variable. Diets were abbreviated as follows: green circles: $0 \%$ concentrate group, orange crosses: $30 \%$ barley concentrate group, red triangles: $60 \%$ barley concentrate group. Color version available online. 


\begin{tabular}{|c|c|c|c|c|c|c|}
\hline Taxonomy & $\begin{array}{l}\text { OTU } \\
\text { No. }\end{array}$ & 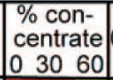 & 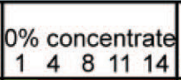 & $\begin{array}{l}30 \% \text { concentrate } \\
25 \\
2\end{array}$ & $\begin{array}{l}60 \% \text { concentrate } \\
3 \quad 6 \quad 10131517\end{array}$ & $\begin{array}{l}\text { Relative } \\
\text { abundance [\%] }\end{array}$ \\
\hline Bergeriella & 1 & & * & $*$ & 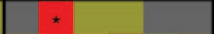 & $19-43$ \\
\hline Campylobacter & 2 & & & & & $18-19$ \\
\hline Campylobacter & 3 & & & * & & $17-18$ \\
\hline Prevotella & 4 & & & & & $16-17$ \\
\hline Bergeriella & 5 & & & & & $15-16$ \\
\hline Prevotella & 6 & & & & & $14-15$ \\
\hline Eikenella & 7 & & & & & $13-14$ \\
\hline Prevotella & 8 & & & & & $12-13$ \\
\hline GNO2 bacterium & 9 & & & & & $11-12$ \\
\hline Treponema & 10 & & & & & $10-11$ \\
\hline Prevotella & 11 & & & & & $9-10$ \\
\hline Treponema & 12 & & & & & $8-9$ \\
\hline Treponema & 13 & & & & & $7-8$ \\
\hline Ottowia & 14 & & & & & $6-7$ \\
\hline Bacteroidetes bacterium & 15 & & & & & $5-6$ \\
\hline Desulfobulbus & 16 & & & & & $4-5$ \\
\hline Ottowia & 17 & & & & & $3-4$ \\
\hline Spirochaeta & 18 & & & & & $2-3$ \\
\hline Clostridiales bacterium & 19 & & & & & $1-2$ \\
\hline SR1 bacterium & 20 & & & & & $>0-1$ \\
\hline Neisseria & 21 & & & & & not detected \\
\hline Bacteroidetes bacterium & 22 & & & & & \\
\hline Desulfobulbus & 23 & & & & & \\
\hline Lachnospiraceae bacterium & 24 & & & & & \\
\hline Mogibacterium & 25 & & & & & \\
\hline Desulfotomaculum & 26 & & & & & \\
\hline Bacteroidetes bacterium & 27 & & & & & \\
\hline Clostridiales bacterium & 28 & & & & & \\
\hline Bacteroidetes bacterium & 29 & & & & & \\
\hline Eikenella & 30 & & & & & \\
\hline Lachnospiraceae bacterium & 31 & & & & & \\
\hline Butyrivibrio & 32 & & & & & \\
\hline Parvibacter & 33 & & & & & \\
\hline Bacteroidetes bacterium & 34 & & & & & \\
\hline Butyrivibrio & 35 & & & & & \\
\hline Butyrivibrio & 36 & & & & & \\
\hline Bacteroidales bacterium & 37 & & & & & \\
\hline Prevotella & 38 & & & & & \\
\hline Deltaproteobacteria bacterium & 39 & & & & & \\
\hline Porphyromonadaceae bacterium & 40 & & & & & \\
\hline Clostridium & 41 & & & & & \\
\hline Porphyromonadaceae bacterium & 42 & & & & & \\
\hline Catonella & 43 & & & & & \\
\hline Eubacterium & 44 & & & & & \\
\hline Succiniclasticum & 45 & & & & & \\
\hline
\end{tabular}

Figure 4. Relative abundance of operational taxonomic units (OTU) per feeding group (0, 30, and $60 \%$ barley concentrate), single goat, and diet-related shifts. Heatmap showing the relative abundances of the 45 most-abundant OTU sorted per feeding group and per individual goat. Diets were abbreviated as follows: A: $0 \%$ concentrate group, B: $30 \%$ concentrate group, and C: $60 \%$ concentrate group. For better resolution, relative abundance values higher than $19 \%$ (ranging from 19 to 43\%) are shown as $19 \%$ and are denoted with an asterisk $(*)$. Color version available online.

The k-means clustering was performed with OTUs that were detected in all 3 feeding groups $(\mathrm{n}=322$, Figure 5). Cluster I consisted of 12 OTU that were highly abundant in the $0 \%$ concentrate feeding group, moderately abundant in the $30 \%$ concentrate feeding group, and scarcely abundant in the $60 \%$ feeding group. These OTUs were classified as Meniscus, Treponema,
Lutispora, unclassified SR1-phylum, Neisseria, Bergeriella, Kingella, Mahella, Butyrivibrio, Paraprevotella, and Sediminitomix. Cluster IV consisted of 62 OTU and contained OTUs that were highly abundant in the $60 \%$ concentrate group and low to moderately abundant in the 2 other feeding groups. These OTUs were classified as Prevotella, Desulfobulbus, Spirochaeta, Guggenhei- 


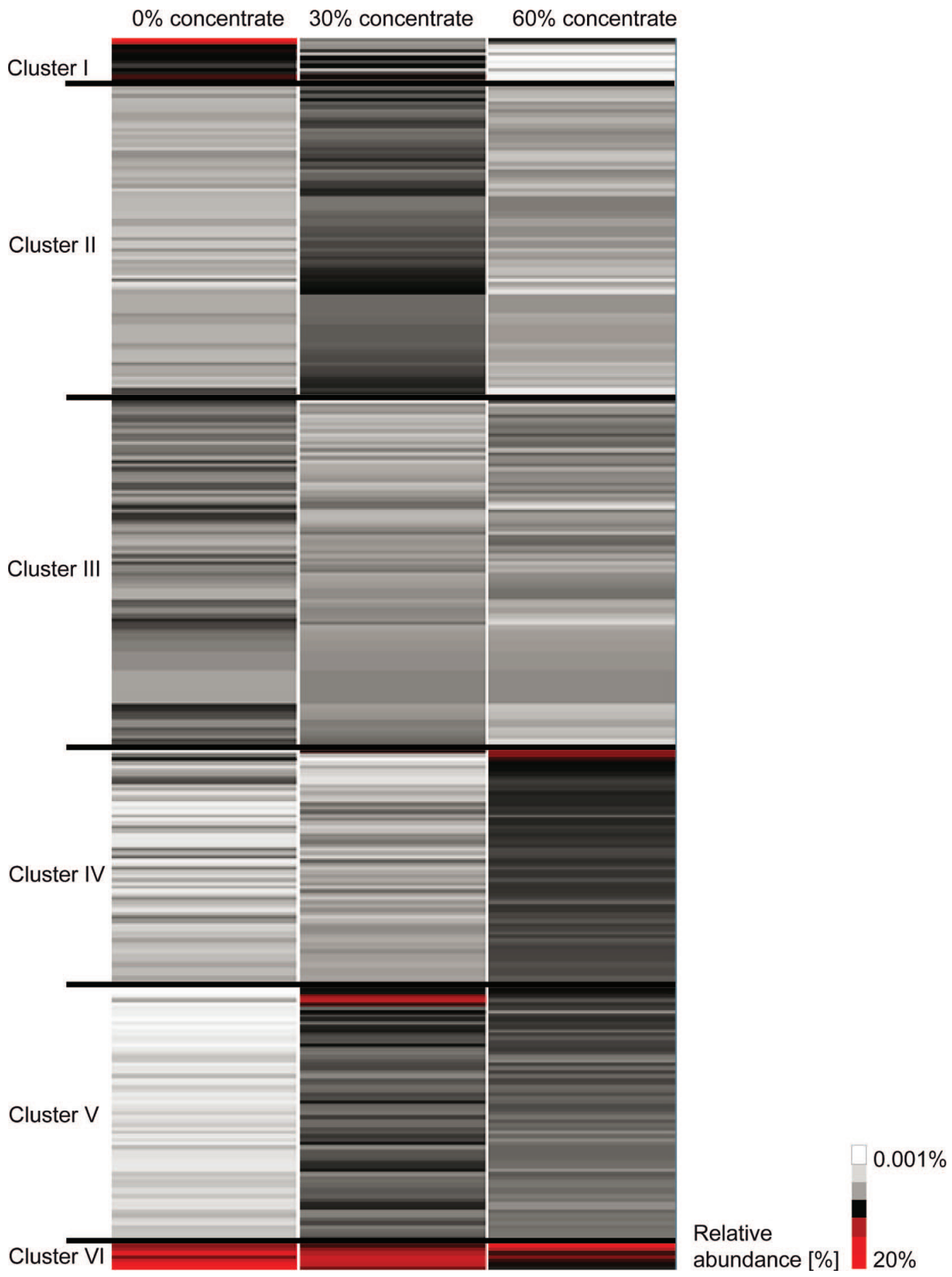

Figure 5. The k-means cluster analysis with operational taxonomic units (OTU) detected in all feeding groups (0,30, and 60\% barley concentrate; $\mathrm{n}=322$ ). Sequences were normalized and the Euclidian distances with mean values were calculated and clustered. Color version available online.

mella, Mogibacterium, Phocaeicola, Butyrivibrio, Howardella, and Butyricimonas. Cluster VI consisted of 8 OTU that were highly abundant in all 3 feeding groups. These OTUs were classified as Bergeriella, Campylobacter, Prevotella, and Paraprevotella.

\section{Comparison of the Roche/454 Pyrosequencing Data Set with Near Full-Length Sequences}

We sequenced 328 16S rRNA gene clones deriving from 3 goats (1 goat from each feeding group) with 
Sanger sequencing and compared them with the pyrosequencing OTUs to obtain better taxonomic resolution of the most abundant OTUs. The OTUs with $\geq 99 \%$ sequence similarity were considered as the same species. In total, 179 OTU of the pyrosequencing data set showed $\geq 99 \%$ sequence similarity with OTUs of the Sanger sequencing data set. In Supplementary Table S2 (http://dx.doi.org/10.3168/jds.2014-9166), the 45 most abundant OTU from the Roche/454 data set and the corresponding near full-length Sanger sequencing matches, if available, are listed.

\section{Metagenome Prediction}

To estimate whether diet might also modify the overall metabolic potential of the CBEM, we applied the recently published bioinformatics tool PICRUSt to the 16S rRNA pyrosequencing data (Langille et al., 2013). The predicted metagenomic structure of CBEM is mostly consistent for animals within one dietary group. The results of this analysis (Supplementary Figure S1; http://dx.doi.org/10.3168/jds.2014-9166) suggested consistency for most metabolic functions, particularly for genes encoding for cellular and information processing, such as the ribosomal machinery or translation and transcription, which do not considerably vary among diets fed. However, clusters of orthologs groups category G (genes for carbohydrate transport and metabolism) and $\mathrm{T}$ (signal transduction mechanisms) were overrepresented in the $60 \%$ concentrate group compared with the other groups (1.3 and 1.2 fold-change, respectively).

\section{Analysis on the Genus Level and Definition of a CBEM Core Microbiome}

In addition to the overview on the phylum and OTU level, we combined all OTUs of the pyrosequencing data set in their respective genus to get insights into cumulated shifts on the genus level. In Figure 6, the potential CBEM core microbiome consisting of the 35 most abundant genera out of 220 genera (genera $>0.003 \%$ relative abundance) is depicted. Relative genera abundances separated per diet are shown in Supplementary Table S5 (http://dx.doi.org/10.3168/ jds.2014-9166). The genera Bergeriella, Campylobacter, and Prevotella were the 3 most abundant genera in all feeding groups and accounted for over $49 \%$ of all sequences. Independent of the feeding group, Bergeriella was the most abundant genus but it represented only the fourth-most abundant genus in the $60 \%$ concentrate group. Goats fed with the $0 \%$ concentrate diet tended to harbor less Ottowia (0.21-fold change, $P<$ 0.07 ) and less Mogibacterium (0.24-fold change, $P<$ 0.03), whereas Kingella increased (5.96-fold change,
$P<0.04)$ compared with goats of the other feeding groups. Furthermore, goats fed the $0 \%$ concentrate diet tended to have more Bergeriella than goats fed the $30 \%$ concentrate diet $(2.1$ fold-change, $P<0.08)$. The genus Bergeriella was significantly decreased (0.22-fold change, $P<0.02)$ in the $60 \%$ feeding group compared with the 0 and $30 \%$ concentrate diets. Feeding of $60 \%$ concentrate diet also tended to increase Prevotella compared with goats fed the 0 and $30 \%$ concentrate diets (1.66 fold-change, $P=0.07$ ).

\section{DISCUSSION}

In general, environments such as the rumen house fastidious bacteria that are difficult to cultivate in vitro. This has led to limited reference databases, which, in turn, hinder the classification of microbes from such complex ecosystems. Molecular techniques such as those employed in our study have demonstrated a far more complex epimural bacterial community in the rumen than expected based on traditional cultivationbased techniques. The advantage of pyrosequencing is the broad coverage of phylotypes, but the phylogenetic resolution for a classification at species level may not be sufficient. For this reason, we additionally sequenced $16 \mathrm{~S}$ rRNA gene clones. Unfortunately, not all of the highly abundant OTUs from pyrosequencing were classified as the same species as one of our near full-length sequences. In near future, the "Hungate1000" project, aiming to sequence reference microbial genomes from the rumen, should greatly help in the characterization of rumen microbes (http://www.hungate1000.org.nz/; Creevey et al., 2014). Until this database is fully developed, it may remain difficult to classify certain rumen bacteria due to the absence of closely related characterized reference strains. The present study revealed striking changes in the CBEM in relation to increasing dietary concentrate levels.

\section{The Bacterial Epimural Microbiome}

One major finding of our study was the high diversity of the CBEM. We can largely exclude a contamination of the rumen mucosa with bacteria originating from the rumen content, because the mucosa was rigorously washed and rinsed during sampling to remove free and loosely attached bacteria. Furthermore, previous studies revealed distinct clustering of the bacterial communities from different rumen fractions, providing evidence for distinct microbial community structures at the 3 rumen fractions (rumen fluid, attached to solid digesta, attached to rumen mucosa; Cunha et al., 2011; Lee et al., 2012; Huo et al., 2014). 


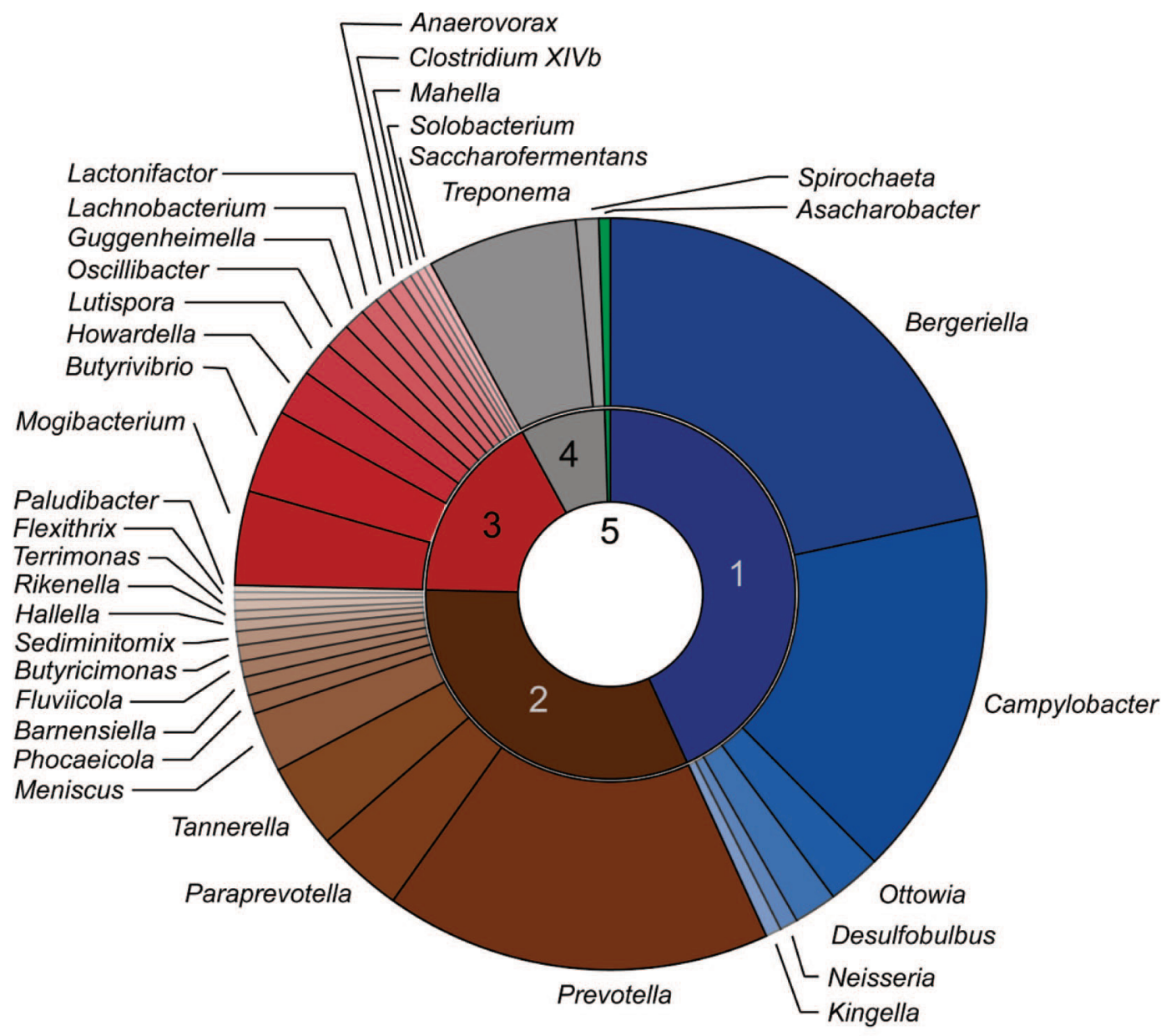

1 - Proteobacteria 2 Bacteroidetes 3 Firmicutes 4 Spirochaetes 5 Actinobacteria

Figure 6. Visualization of the caprine bacterial epimural microbiome core on genus and phylum level, independent of the goat diet. The most abundant genera over all feeding groups $(0,30$, and $60 \%$ barley concentrate) are shown. The size of the circle fragments is proportional to the number of sequences. Color version available online.

A feeding experiment with different energy supplies revealed Firmicutes, Bacteroidetes, Chloroflexi, Lentisphaerae, Proteobacteria, Spirochaetes, and Tenericutes to be the most abundant phyla in the liquid and solid fraction of the rumen of goats using Roche/454 pyrosequencing (Huo et al., 2014). Also, the bacterial community in the liquid- and solid-associated fractions of the rumen digesta of goats was described using $16 \mathrm{~S}$ rRNA gene libraries (Cunha et al., 2011). The phyla Bacteroidetes and Firmicutes were predominant, whereas Proteobacteria were only observed in the liquid fraction $(0.5 \%)$ and Spirochaetes were not found. The results of these 2 studies are in contrast to our study, in which we found a high abundance of Proteobacteria. Despite all of these studies being conducted on goats, the different results obtained may indicate differences between the luminal core microbiome and CBEM in the rumen. Recently, Liu et al. (2015) also found the phyla Firmicutes,
Bacteroidetes, and Proteobacteria to be most abundant in the CBEM and increasing Firmicutes with highconcentrate diet, which is consistent with our results. Mogibacterium increased with increasing concentrate amount in both studies. Mogibacterium are known to be nonfermentative, are not nitrate-reducers, and were isolated from human periodontal pockets (Nakazawa et al., 2000); thus, it is difficult to predict their metabolic role in the CBEM. However, due to the different sampling methods, DNA isolation methods, primers, data processing methods, and because different breeds were used, it is hard to compare their study with our study.

In our study, we expected Proteobacteria to decrease and Firmicutes to increase with increasing concentrate amount because of the susceptibility of many Proteobacteria toward low $\mathrm{pH}$ (Rousk et al., 2010) and the ability of many Firmicutes to degrade easily fermentable carbohydrates, even under low $\mathrm{pH}$. Previous studies al- 
ready noted such shifts in the microbiome of the rumen epithelium in other ruminant species fed with different energy levels (Chen et al., 2011; Li et al., 2012; Petri et al., 2013a). This leads to the hypothesis that some parts of the core microbiome may be shared among different ruminants and may behave in a similar way to different feeding strategies. Interestingly, many of the OTUs obtained in our study showed highest similarity $(>99 \%)$ to sequences originating from cattle and sheep rumen samples (Supplementary Table S6; http:// dx.doi.org/10.3168/jds.2014-9166; Sadet-Bourgeteau et al., 2010; Li et al., 2012), which also supports the existence of a potential epimural ruminant core microbiome.

However, Proteobacteria could also be triggered by the presence of trace amounts of oxygen, as many organisms belonging to this phylum are microaerophilic or facultative anaerobes (Sadet-Bourgeteau et al., 2010). Bacteria belonging to Firmicutes often carry more than 4 rRNA operons, even up to 15 operons, which may have led to an overestimation of the Firmicutes abundance (Lee et al., 2009).

At the genus level, our study reveals the most abundant genus to be classified as Bergeriella. Bergeriella is a nonflagellated commensal, which is known to effectively ferment carbohydrates from its host. Bergeriella have rarely been described in rumen samples before (Pallara et al., 2014), but it is well known as a nonpathogen in oral cavities of different mammals (Xie and Yokota, 2005). Unlike Neisseria species, it can produce fermentation acids from mannose, glucose, fructose, and sucrose, which may also lead to a decrease of rumen $\mathrm{pH}$. The nitrite-reducing properties of Neisseria species might be helpful for the host ruminant to avoid nitrate poisoning (Lewis, 1951; Kahn, 2005). Pathogenic relatives of Bergeriella, such as Neisseria gonorrhoeae, Neisseria meningitides, or Neisseria ovis, are associated with gonorrhea, meningitis, and conjunctivitis in ruminants, respectively (Bankemper et al., 1990). Considering the high abundance of Neisseriaceae in our study with goats being clinically healthy, we do not expect them to have pathogenic effect on the rumen, but until now it is unclear whether it may have zoonosis relevance. Due to the low sequence similarity (92\% for OTU1 and $91 \%$ for OTU5), we cannot exclude that these OTUs may belong to a different genus than Bergeriella. Pyrosequencing OTUs classified as Bergeriella showed highest similarity to Snodgrassella alvi (94\% similarity, BlastN against GenBank nr), a carboxylic acid-oxidizing dominant member of the honey bee and bumble bee digestive tract (Kwong et al., 2014). Kwong et al. (2014) stated that Snodgrassella alvi may form a syntrophic network for sharing metabolic resources with other species. Therefore, we hypothesized that OTUs classified as Bergeriella may have a similar function in the goat rumen epithelium.

Campylobacter, the second-most prevalent genus detected, showed no significant difference in response to different concentrate supply. Known pathogens in this genus are Campylobacter jejuni and Campylobacter coli, which cause enteritis and are common in the intestines of mammals and birds. Further studies are needed to reliably classify Campylobacter on species level and to identify whether they represent commensals or potentially pathogens in the caprine rumen (Garrity, 2005; Anderson et al., 2009; Lin et al., 2013).

Prevotella was the third-most abundant genus in our study and is commonly known to be an abundant member of the rumen microbiome (Malmuthuge et al., 2014; Omoniyi et al., 2014; Rosewarne et al., 2014). They have highly diverse functions and are one of the most abundant genera in the rumen content of goats, in particular after high-grain feeding (Khafipour et al., 2009; Metzler-Zebeli et al., 2013b). Correlation analysis showed positive relationships between Prevotella, ruminal LPS concentration (Tamura et al., 1992), colonic LPS concentration, and ruminal SCFA concentration (Metzler-Zebeli et al., 2013b). In our study, some Prevotella-OTU increased whereas others decreased between different concentrate feeding groups, finally causing no statistically significant shifts on genus level. Due to the high diversity of Prevotella species and their metabolic functions, we cannot predict their exact metabolic function. Some Prevotella might shed high amounts of LPS, which can float into the blood circuit of the goat and cause systemic inflammation reactions (Khafipour et al., 2009). Second, Prevotella species producing SCFA as end products could possibly play an important role in the rumen of goats fed high-energy supply diets, as SCFA are important substrates in the rumen metabolism (Kristensen et al., 1998). Under acidic conditions, high concentrations of SCFA lead to a decrease of ruminal $\mathrm{pH}$, causing epithelial lesions in the rumen wall (Zebeli and Metzler-Zebeli, 2012).

\section{Diet-Related Effects and Metabolic Prediction}

Increasing the concentrate level of the diet had a substantial effect on the bacterial community attached to the mucosa, as indicated by the trend for an increased species richness and Firmicutes abundance in the $60 \%$ concentrate group compared with the 2 other groups. Distinct clustering per feeding group in the Bray-Curtis similarity matrix provided strong evidence for distinct microbial communities due to different concentrate amounts confirming our hypothesis. Although all goats grew up in the same barn, distinct community patterns were found within feeding groups (shown by 
Bray-Curtis analysis), suggesting an individual CBEM with a shared core microbiome of abundant community members. In a recent study, shifts in the potential luminal goat rumen microbiome due to different energy supply were detected (Huo et al., 2014). The difference in bacterial communities between the $60 \%$ concentrate group and the other 2 feeding groups could be due to the strong effect of a decrease in rumen $\mathrm{pH}$, which could lead to rising fermentation and alterations in rumen mucosal functioning. An increase of osmolarity with high concentrate levels fed (Hollmann et al., 2013; Metzler-Zebeli et al., 2013a) may cause increased water influx into the rumen and decrease barrier function of the epithelium (Penner, 2009). Increased water influx into the rumen may be one reason for feed intake depression during SARA in ruminants, which has a negative effect on animal health as well as on dairy production. With high-concentrate diets, rumen conditions are comparable to conditions observed during SARA. For example, ruminal $\mathrm{pH}$ was 5.5 and 6.0 in the 60 and $30 \%$ concentrate groups, respectively. In cattle, ruminal $\mathrm{pH}$ values below 5.8 are typical SARA thresholds (Zebeli and Metzler-Zebeli, 2012), whereas in goats no such ruminal $\mathrm{pH}$ thresholds have yet been characterized. Subacute ruminal acidosis is known to provoke greater metabolic stress of microbiota and lower overall fiber digestion. A high number of lactate-producers and disturbed mucosal absorption of SCFA often precede SARA (Zebeli and Metzler-Zebeli, 2012). The cluster analysis revealed nearly half of all OTU abundances were hardly shifted in the $0 \%$ concentrate feeding group compared with the $60 \%$ concentrate feeding group, which indicates the fraction of relatively stable OTU when a high-concentrate diet is fed. Klevenhusen et al. (2013) analyzed the same goats with respect to altered electrophysiological properties and permeability of the rumen wall. The $\mathrm{pH}$ of the rumen was measured directly after euthanasia $(2-3 \mathrm{~h}$ after last morning feeding). Although the values of the ruminal $\mathrm{pH}$ during the feeding experiment are not known, we can assume that at least the $60 \%$ concentrate feeding group $(\mathrm{pH}$ 5.5) have been at high risk of SARA (Klevenhusen et al., 2013).

The OTUs belonging to cluster I (k-means cluster analysis), highly abundant in the $0 \%$ concentrate feeding group and low to moderately abundant in the other 2 feeding groups, are expected to mostly ferment cellulose and be involved in fiber digestion. These OTUs, or their close relatives, are common in the gastrointestinal tract of different mammals and ferment different cell-wall carbohydrates to acetic acid and interact with cellulolytic bacteria or are cellulolytic themselves (Stanton and Canale-Parola, 1980; Rumney et al., 1995). Lutispora ferments peptone, tryptone, caseine, pyruvate, and some AA and produces acetate, isobutyrate, propionate, and isovalerate (Shiratori et al., 2008). Mahella can ferment various plant disaccharides and simple sugars such as arabinose, cellobiose, fructose, glucose, sucrose, xylose, and pyruvate to lactate and formate (Salinas et al., 2004). The accumulation of these OTUs in the $0 \%$ concentrate group underlines the importance of these bacteria for cellulose fermenting processes. The most abundant OTUs in cluster IV, highly abundant OTUs in the $60 \%$ concentrate group, are mostly responsible for different fermentation processes (e.g., cellulolytic, hemicellulolytic, oligosaccharolytic, proteolytic, amylolytic, and xylanolytic functions) or are not involved in fermentation processes at all (Nakazawa et al., 2000). Prevotella is also known to increase with decreasing rumen $\mathrm{pH}$ (Petri et al., 2013b). Desulfobulbus is a sulfate-reducer that also decomposes FA (Widdel and Pfennig, 1982). Butyrivibrio ferments glucose to butyrate, hydrogen, ethanol, carbon dioxide, formate, and lactate (Kopecný et al., 2003). According to the literature, most of these OTUs are involved in the fermentation of easily fermentable carbohydrates or SCFA, as their concentration in the rumen increases with increasing energy supply (Petri et al., 2013a; Huo et al., 2014). The OTUs in cluster VI, highly abundant OTUs in all 3 feeding groups, ferment different carbohydrates (such as cellulose, hemicellulose, saccharose, and others) to acetic acid and other metabolites, interact with cellulolytic bacteria and are cellulolytic themselves (Petri et al., 2013b), or reduce nitrate (Vedros et al., 1983). Campylobacter is a nitrate- and sulfite-reducing (except C. jejuni), fumarate to succinate reducing, asaccharolytic bacterium relying on fermentation of AA or tricarboxylic acids (Garrity, 2005). All of these phylotypes may also additionally be involved in cross-feeding processes, which might explain their high abundance although their favorite substrate is not known to be produced in high amounts. It is, however, hard to predict which metabolic influence these bacteria have on the host because some of the OTUs showed only low similarity $(<90 \%)$ to their closest related type strains.

To obtain insight into the putative functional capacity of the CBEM, PICRUSt (Langille et al., 2013) was applied. This tool was used recently for the prediction of gene families and was shown to reliably outline metagenomic approaches from the Human Microbiome Project (Langille et al., 2013). The PICRUSt analysis generated 2 hypotheses: (1) goats have a stable functional metagenome of the CBEM that is hardly influenced by the concentrate percentage in the diet and (2) signal transduction, defense, and carbohydrate metabolism are increased in the $60 \%$ concentrate group. This increased carbohydrate metabolism can be 
explained by the fact that the $60 \%$ concentrate group is associated with a higher amount of easily fermentable carbohydrates in the rumen which serve as easily available energy substrates for rumen CBEM, leading to an adaptation of the carbohydrate metabolism in the rumen epithelium too. The high concentration of easily fermentable carbohydrates and metabolic end products may cause increased osmolarity at the mucosal site. The increased water influx into the rumen can cause damage to the epithelial barrier function and thus foster translocation of pathogens into the blood stream. Although we observed downregulation of several protective proteins in the rumen epithelium due to $60 \%$ grain feeding (Hollmann et al., 2013), and also an increase in the permeability of rumen wall (Klevenhusen et al., 2013), the concentration of serum amyloid A did not differ in our study (Klevenhusen et al., 2013). An increase in serum amyloid A would have been a hint for activation of acute-phase response due to endotoxin translocation, which is observed during episodes of SARA (Zebeli and Metzler-Zebeli, 2012). We assume that goats of our study did experience a severe form of SARA with systemic inflammation, as observed in cattle fed similar grain-rich diets (Khafipour et al., 2009). Additionally these goats also showed increased papillae lesion score, increased keratinization score, decreased fecal score, and increased pulse and respiration rates (Klevenhusen et al., 2013; Metzler-Zebeli et al., 2013b).

\section{CONCLUSIONS}

Interestingly, many of the OTUs obtained in our study showed the highest similarity to sequences originating from cattle and sheep rumen samples, which supports the hypothesis of some analogies between microbiomes of different ruminants. The results demonstrate dynamics in the CBEM, triggered by high-concentrate feeding, and revealed distinct microbial communities for all 3 diets tested. This confirms the general hypothesis that diet has a big effect on microbial communities in the gastrointestinal tract. Fiber-digesting bacteria decreased whereas starch-fermenting bacteria were enhanced in the high-concentrate feeding group. These shifts underline the severe risk of SARA in ruminants, as it is not only relevant in cattle, but also in goats. Goat dairy and meat production can be negatively affected by inadequate feeding strategies. Collectively, the observed microbial findings may have important relevance in goat-production systems, whose feeding practices are increasingly changing from traditional roughage-based to concentrate-rich diets to support higher production levels. Future work will be important for the characterization of the CBEM to unravel the function of microbes under defined ruminal conditions and for defining indicator species for the prediction of rumen disorders.

\section{ACKNOWLEDGMENTS}

The study was funded by Vienna Science and Technology Fund (WWTF, Vienna, Austria) through project "D-i.INFLACOW, LS12-010" led by Q. Zebeli and by the science funds of the Land Niederoesterreich, Austria (project ID: MIKRORIND, St. Pölten, Austria). The support of Anneliese Müller (Institute for Milk Hygiene, Milk Technology and Food Science, University of Veterinary Medicine Vienna, Austria) with laboratory work is thankfully acknowledged. We are grateful to H. Weissenböck and the team of the Institute of Pathology and Forensic Veterinary Medicine, University of Veterinary Medicine Vienna, and also to Stefanie Burger and the Institute of Anatomy, Histology and Embryology, Department Pathophysiology, University of Veterinary Medicine Vienna, for their help. We also are thankful to L. Podstatzky-Lichtenstein and the staff of the Institute for Organic Farming and Bioderversity (LFZ Raumberg-Gumpenstein, Wels, Austria) and to R. Krametter-Frötscher (Clinic for Ruminants, University of Veterinary Medicine Vienna) for caring of the experimental goats. The help of A. Dockner, M. Wild, C. von Reitzenstein, and R. Tienken (Institute of Animal Nutrition and Functional Plant Compounds, University of Veterinary Medicine Vienna) during the experiment and Monika Dzieciol (Institute for Milk Hygiene, Milk Technology and Food Science, University of Veterinary Medicine) and Corinne Stouthamer (Department of Entomology, University of Arizona, Tucson) is also greatly acknowledged.

\section{REFERENCES}

Amann, R. I., W. Ludwig, and K. H. Schleifer. 1995. Phylogenetic identification and in situ detection of individual microbial cells without cultivation. Microbiol. Rev. 59:143-169.

Anderson, R. C., N. A. Krueger, J. A. Byrd, R. B. Harvey, T. R. Callaway, T. S. Edrington, and D. J. Nisbet. 2009. Effects of thymol and diphenyliodonium chloride against Campylobacter spp. during pure and mixed culture in vitro. J. Appl. Microbiol. 107:1258-1268.

Bankemper, K. W., D. M. Lindley, K. E. Nusbaum, and R. H. Mysinger. 1990. Keratoconjunctivitis associated with Neisseria ovis infection in a herd of goats. J. Vet. Diagn. Invest. 2:76-78.

Bergman, E. N. 1990. Energy contributions of volatile fatty-acids from the gastrointestinal tract in various species. Physiol. Rev. 70:567-590.

Chen, Y., G. B. Penner, M. Li, M. Oba, and L. L. Guan. 2011. Changes in bacterial diversity associated with epithelial tissue in the beef cow rumen during the transition to a high-grain diet. Appl. Environ. Microbiol. 77:5770-5781.

Cheng, K. J., and R. J. Wallace. 1979. The mechanism of passage of endogenous urea through the rumen wall and the role of ureolytic epithelial bacteria in the urea flux. Br. J. Nutr. 42:553-557. 
Creevey, C. J., W. J. Kelly, G. Henderson, and S. C. Leahy. 2014 Determining the culturability of the rumen bacterial microbiome. Microb. Biotechnol. 7:467-479.

Cunha, I. S., C. C. Barreto, O. Y. A. Costa, M. A. Bomfim, A. P. Castro, and R. H. Kruger., and BQuirino, F. 2011. Bacteria and archaea community structure in the rumen microbiome of goats (Capra hircus) from the semiarid region of Brazil. Anaerobe $17: 118-124$.

de Hoon, M. J., S. Imoto, J. Nolan, and S. Miyano. 2004. Open source clustering software. Bioinformatics 20:1453-1454.

Dehority, B. A., and J. A. Grubb. 1977. Characterization of the predominant bacteria occurring in the rumen of goats (Capra hircus). Appl. Environ. Microbiol. 33:1030-1036.

Fernando, S. C., H. T. Purvis, F. Z. Najar, L. O. Sukharnikov, C. R. Krehbiel, T. G. Nagaraja, B. A. Roe, and U. DeSilva. 2010. Rumen microbial population dynamics during adaptation to a highgrain diet. Appl. Environ. Microbiol. 76:7482-7490.

Garrity, G. M. 2005. Bergey's Manual of Systematic Bacteriology. Vol. 2. The Proteobacteria Part C: The Alpha-, Beta-, Delta-, and Epsilonproteobacteria No. 2. Springer, New York, NY.

Griffen, A. L., C. J. Beall, J. H. Campbell, N. D. Firestone, P. S. Kumar, Z. K. Yang, M. Podar, and E. J. Leys. 2012. Distinct and complex bacterial profiles in human periodontitis and health revealed by $16 \mathrm{~S}$ pyrosequencing. ISME J. 6:1176-1185.

Han, X., Y. Yang, H. Yan, X. Wang, L. Qu, and Y. Chen. 2015. Rumen bacterial diversity of 80 to 110-day-old goats using $16 \mathrm{~S}$ rRNA sequencing. PLoS ONE 10:e117811.

Hollmann, M., I. Miller, K. Hummel, S. Sabitzer, B. U. Metzler-Zebeli, E. Razzazi-Fazeli, and Q. Zebeli. 2013. Downregulation of cellular protective factors of rumen epithelium in goats fed high energy diet. PLoS ONE 8:e81602.

Hungate, R. E. 1966. The Rumen and its Microbes. Academic Press Inc., New York, NY.

Huo, W., W. Zhu, and S. Mao. 2014. Impact of subacute ruminal acidosis on the diversity of liquid and solid-associated bacteria in the rumen of goats. World J. Microbiol. Biotechnol. 30:669-680.

Joachimiak, M. P., J. L. Weisman, and B. May. 2006. JColorGrid: Software for the visualization of biological measurements. BMC Bioinformatics 7:225.

Juretschko, S., G. Timmermann, M. Schmid, K. H. Schleifer, A. Pommerening-Röser, H. P. Koops, and M. Wagner. 1998. Combined molecular and conventional analyses of nitrifying bacterium diversity in activated sludge: Nitrosococcus mobilis and Nitrospiralike bacteria as dominant populations. Appl. Environ. Microbiol. 64:3042-3051.

Kahn, C. M. L. S. 2005. Toxicology: Nitrate and nitrite poisoning. Pages 2423-2426 in The Merck Veterinary Manual. 9th ed. S. E. Aiello and M. A. Moses, ed. Merck, Whitehouse Station, NJ.

Kamra, D. N. 2005. Rumen microbial ecosystem. Curr. Sci. 89:124135.

Khafipour, E., S. C. Li, J. C. Plaizier, and D. O. Krause. 2009. Rumen microbiome composition determined using two nutritional models of subacute ruminal acidosis. Appl. Environ. Microbiol. 75:7115-7124.

Klevenhusen, F., M. Hollmann, L. Podstatzky-Lichtenstein, R. Krametter-Frotscher, J. R. Aschenbach, and Q. Zebeli. 2013. Feeding barley grain-rich diets altered electrophysiological properties and permeability of the ruminal wall in a goat model. J. Dairy Sci. 96:2293-2302.

Kopecný, J., M. Zorec, J. Mrazek, Y. Kobayashi, and R. MarinsekLogar. 2003. Butyrivibrio hungatei sp. nov. and Pseudobutyrivibrio xylanivorans sp. nov., butyrate-producing bacteria from the rumen. Int. J. Syst. Evol. Microbiol. 53:201-209.

Kristensen, N. B., A. Danfaer, and N. Agergaard. 1998. Absorption and metabolism of short chain fatty acids in ruminants. Arch. Tierernahr. 51:165-175.

Kuczynski, J., C. L. Lauber, W. A. Walters, L. W. Parfrey, J. C. Clemente, D. Gevers, and R. Knight. 2012. Experimental and analytical tools for studying the human microbiome. Nat. Rev. Genet. $13: 47-58$.
Kwong, W. K., P. Engel, H. Koch, and N. A. Moran. 2014. Genomics and host specialization of honey bee and bumble bee gut symbionts. Proc. Natl. Acad. Sci. USA 111:11509-11514.

Lane, D. J. 1991. 16S/23S rRNA Sequencing. Nucleic Acid Techniques in Bacterial Systematics. Wiley, New York, NY.

Langille, M. G. I., J. Zaneveld, J. G. Caporaso, D. McDonald, D. Knights, J. A. Reyes, J. C. Clemente, D. E. Burkepile, R. L. V. Thurber, R. Knight, R. G. Beiko, and C. Huttenhower. 2013. Predictive functional profiling of microbial communities using $16 \mathrm{~S}$ rRNA marker gene sequences. Nat. Biotechnol. 31:814-821.

Lee, H. J., J. Y. Jung, Y. K. Oh, S. S. Lee, E. L. Madsen, and C. O. Jeon. 2012. Comparative survey of rumen microbial communities and metabolites across one caprine and three bovine groups, using bar-coded pyrosequencing and ${ }^{1} \mathrm{H}$ nuclear magnetic resonance spectroscopy. Appl. Environ. Microbiol. 78:5983-5993.

Lee, Z. M., C. Bussema 3rd, and T. M. Schmidt. 2009. rrnDB: Documenting the number of rRNA and tRNA genes in bacteria and archaea. Nucleic Acids Res. 37:D489-D493.

Leser, T. D., and L. Molbak. 2009. Better living through microbial action: The benefits of the mammalian gastrointestinal microbiota on the host. Environ. Microbiol. 11:2194-2206.

Lewis, D. 1951. The metabolism of nitrate and nitrite in the sheep: The reduction of nitrate in the rumen of the sheep. Biochem. J. $48: 175-180$.

Li, M., M. Zhou, E. Adamowicz, J. A. Basarab, and L. L. Guan. 2012. Characterization of bovine ruminal epithelial bacterial communities using $16 \mathrm{~S}$ rRNA sequencing, PCR-DGGE, and qRT-PCR analysis. Vet. Microbiol. 155:72-80.

Lin, M., W. Guo, Q. Meng, D. M. Stevenson, P. J. Weimer, and D. M. Schaefer. 2013. Changes in rumen bacterial community composition in steers in response to dietary nitrate. Appl. Microbiol. Biotechnol. 97:8719-8727.

Liu, J. H., G. Bian, W. Zhu, and S. Mao. 2015. High-grain feeding causes strong shifts in ruminal epithelial bacterial community and expression of Toll-like receptor genes in goats. Front. Microbiol. $6: 167$.

Luckey, T. D. 1972. Introduction to intestinal microecology. Am. J. Clin. Nutr. 25:1292-1294.

Mackie, R. I. 2000. Molecular ecology and diversity in gut microbial ecosystems. Pages 61-77 in Ruminant Physiology: Digestion, Metabolism, Growth and Reproduction. P. B. Cronje, ed. CABI, Wallingford, UK

Malmuthuge, N., P. J. Griebel, and L. Guan le. 2014. Taxonomic identification of commensal bacteria associated with the mucosa and digesta throughout the gastrointestinal tracts of preweaned calves. Appl. Environ. Microbiol. 80:2021-2028.

Metzler-Zebeli, B. U., M. Hollmann, S. Sabitzer, L. Podstatzky-Lichtenstein, D. Klein, and Q. Zebeli. 2013a. Epithelial response to high-grain diets involves alteration in nutrient transporters and $\mathrm{Na}^{+} / \mathrm{K}^{+}$-ATPase mRNA expression in rumen and colon of goats. J. Anim. Sci. 91:4256-4266.

Metzler-Zebeli, B. U., S. Schmitz-Esser, F. Klevenhusen, L. Podstatzky-Lichtenstein, M. Wagner, and Q. Zebeli. 2013b. Grain-rich diets differently alter ruminal and colonic abundance of microbial populations and lipopolysaccharide in goats. Anaerobe 20:65-73.

Nakazawa, F., M. Sato, S. E. Poco, T. Hashimura, T. Ikeda, S. Kalfas, G. Sundqvist, and E. Hoshino. 2000. Description of Mogibacterium pumilum gen. nov., sp nov and Mogibacterium vescum gen. nov., sp nov., and reclassification of Eubacterium timidum (Holdeman et al 1980) as Mogibacterium timidum gen. nov., comb. nov. Int. J. Syst. Evol. Microbiol. 50:679-688.

Omoniyi, L. A., K. A. Jewell, O. A. Isah, A. P. Neumann, C. F. Onwuka, O. M. Onagbesan, and G. Suen. 2014. An analysis of the ruminal bacterial microbiota in West African Dwarf sheep fed grass- and tree-based diets. J. Appl. Microbiol. 116:1094-1105.

Pallara, G., A. Buccioni, R. Pastorelli, S. Minieri, M. Mele, S. Rapaccini, A. Messini, M. Pauselli, M. Servili, L. Giovannetti, and C. Viti. 2014. Effect of stoned olive pomace on rumen microbial communities and polyunsaturated fatty acids biohydrogenation: An in vitro study. BMC Vet. Res. 10:271. 
Penner, G. B. 2009. Mucosal acidification and hyperosmolarity differentially affect the barrier function of the isolated ovine ruminal epithelia. In Ruminant Physiology. Wageningen Academic Publishers, Wageningen, the Netherlands.

Petri, R. M., T. Schwaiger, G. B. Penner, K. A. Beauchemin, R. J. Forster, J. J. McKinnon, and T. A. McAllister. 2013a. Changes in the rumen epimural bacterial diversity of beef cattle as affected by diet and induced ruminal acidosis. Appl. Environ. Microbiol. 79:3744-3755.

Petri, R. M., T. Schwaiger, G. B. Penner, K. A. Beauchemin, R. J. Forster, J. J. McKinnon, and T. A. McAllister. 2013b. Characterization of the core rumen microbiome in cattle during transition from forage to concentrate as well as during and after an acidotic challenge. PLoS ONE 8:e83424.

Rosewarne, C. P., P. B. Pope, J. L. Cheung, and M. Morrison. 2014. Analysis of the bovine rumen microbiome reveals a diversity of Sus-like polysaccharide utilization loci from the bacterial phylum Bacteroidetes. J. Ind. Microbiol. Biotechnol. 41:601-606.

Rousk, J., E. Baath, P. C. Brookes, C. L. Lauber, C. Lozupone, J. G. Caporaso, R. Knight, and N. Fierer. 2010. Soil bacterial and fungal communities across a $\mathrm{pH}$ gradient in an arable soil. ISME J. 4:1340-1351.

Rumney, C. J., S. H. Duncan, C. Henderson, and C. S. Stewart. 1995. Isolation and characteristics of a wheatbran-degrading Butyrivibrio from human feces. Lett. Appl. Microbiol. 20:232-236.

Sadet, S., C. Martin, B. Meunier, and D. P. Morgavi. 2007. PCR-DGGE analysis reveals a distinct diversity in the bacterial population attached to the rumen epithelium. Animal 1:939-944.

Sadet-Bourgeteau, S., C. Martin, and D. P. Morgavi. 2010. Bacterial diversity dynamics in rumen epithelium of wethers fed forage and mixed concentrate forage diets. Vet. Microbiol. 146:98-104.

Saldanha, A. J. 2004. Java Treeview-Extensible visualization of microarray data. Bioinformatics 20:3246-3248.

Salinas, M. B., M. L. Fardeau, P. Thomas, J. L. Cayol, B. K. Patel, and B. Ollivier. 2004. Mahella australiensis gen. nov., sp. nov., a moderately thermophilic anaerobic bacterium isolated from an Australian oil well. Int. J. Syst. Evol. Microbiol. 54:2169-2173.

Schloss, P. D., and S. L. Westcott. 2011. Assessing and improving methods used in operational taxonomic unit-based approaches for 16S rRNA gene sequence analysis. Appl. Environ. Microbiol. $77: 3219-3226$.

Schloss, P. D., S. L. Westcott, T. Ryabin, J. R. Hall, M. Hartmann, E. B. Hollister, R. A. Lesniewski, B. B. Oakley, D. H. Parks, C. J. Robinson, J. W. Sahl, B. Stres, G. G. Thallinger, D. J. Van Horn, and C. F. Weber. 2009. Introducing mothur: Open-source, platform-independent, community-supported software for describing and comparing microbial communities. Appl. Environ. Microbiol. 75:7537-7541

Shiratori, H., H. Ohiwa, H. Ikeno, S. Ayame, N. Kataoka, A. Miya, T. Beppu, and K. Ueda. 2008. Lutispora thermophila gen. nov., sp. nov., a thermophilic, spore-forming bacterium isolated from a thermophilic methanogenic bioreactor digesting municipal solid wastes. Int. J. Syst. Evol. Microbiol. 58:964-969.

Stanton, T. B., and E. Canale-Parola. 1980. Treponema bryantii sp. nov., a rumen spirochete that interacts with cellulolytic bacteria. Arch. Microbiol. 127:145-156.

Tamura, M., M. Tokuda, S. Nagaoka, and H. Takada. 1992. Lipopolysaccharides of Bacteroides-intermedius (Prevotella intermedia) and Bacteroides (Porphyromonas) gingivalis induce interleukin-8 geneexpression in human gingival fibroblast-cultures. Infect. Immun. 60:4932-4937.

Vedros, N. A., C. Hoke, and P. Chun. 1983. Neisseria-macacae sp-nov, a new Neisseria species isolated from the oropharynges of rhesusmonkeys (Macaca-Mulatta). Int. J. Syst. Bacteriol. 33:515-520.

Widdel, F., and N. Pfennig. 1982. Studies on dissimilatory sulfatereducing bacteria that decompose fatty-acids. 2 . Incomplete oxidation of propionate by Desulfobulbus propionicus gen-nov, sp-nov. Arch. Microbiol. 131:360-365.

Xie, C. H., and A. Yokota. 2005. Phylogenetic analysis of Alysiella and related genera of Neisseriaceae: proposal of Alysiella crassa comb. nov., Conchiformibium steedae gen. nov., comb. nov., Conchiformibium kuhniae sp. nov. and Bergeriella denitrificans gen. nov., comb. nov. J. Gen. Appl. Microbiol. 51:1-10.

Zebeli, Q., and B. U. Metzler-Zebeli. 2012. Interplay between rumen digestive disorders and diet-induced inflammation in dairy cattle. Res. Vet. Sci. 93:1099-1108. 